\title{
Original
}

\section{Evaluation of Regenerative Processes in a Rat Model of Mandibular Condyle Defect using in vivo Micro X-Ray Computed Tomography}

\author{
Tadahito Saito $^{1,2,4)}$, Takayuki Mashimo ${ }^{1,2)}$, Hiroshi Shiratsuchi ${ }^{1,2)}$, Shunsuke Namaki', \\ Kunihito Matsumoto ${ }^{3)}$, Yoshiyuki Mori ${ }^{4)}$, Toru Ogasawara ${ }^{4)}$, Yoshinori Arai ${ }^{5}$, \\ Kazuya Honda ${ }^{3,6}$ and Yoshiyuki Yonehara ${ }^{2,7)}$
}

\author{
1) Division of Oral Structural and Functional Biology Nihon University Graduate School of Dentistry, Tokyo, Japan \\ 2) Department of Oral and Maxillofacial Surgery, Nihon University School of Dentistry, Tokyo, Japan \\ 3) Department of Oral and Maxillofacial Radiology, Nihon University School of Dentistry, Tokyo, Japan \\ 4) Department of Oral and Maxillofacial Surgery, The University of Tokyo Hospital, Tokyo, Japan \\ ${ }^{5)}$ Nihon University School of Dentistry, Tokyo Japan \\ 6) Division of Advanced Dental Treatment, Dental Research Center, Nihon University School of Dentistry, Tokyo, Japan \\ 7) Division of Systemic Biology and Oncology, Dental Research Center, Nihon University School of Dentistry, Tokyo, Japan \\ (Accepted for publication, June 20, 2012)
}

\begin{abstract}
To determine whether the periosteum influences mandibular head regeneration after condylectomy in rats. male Wistar rats, the periosteum of part of the mandibular head was preserved or removed and the mandibular head was excised up to a section of the neck of the mandible. Radiological and histological findings were evaluated $0,1,2,3,4,6$, and 8 weeks later.

Although bone regeneration was observed during the first postoperative week in both groups, restoration occurred from the bone stump and from a position distant from the bone stump when the periosteum was conserved. When the periosteum was removed, restoration occurred from the bone stump only. Regenerated bone mass was greater with the preserved periosteum.

Bone mass increased until the sixth week after condylectomy in the periosteum preservation group. Our results confirm the importance of the periosteum in mandibular head regeneration after condylectomy in rats.
\end{abstract}

Keywords: Bone regeneration, Periosteum, Condylectomy, Micro CT

\section{Introduction}

The temporomandibular joint, which is the only movable joint structure in the maxillofacial region, is an important site for movement associated with ingestion and speaking. The mandibular condylar process is a component of the temporomandibular joint, and it may be transformed by surgery for fractures, inflammation, tumors, and tumor transection. The form of the mandible changes as transformation occurs; this further influences temporomandibular movement and may cause dysfunctions in the occlusion state and speaking or dysphagia.

Procedures such as bone grafting, osteotomy, and condylectomy have been performed in glenoid transformations. Although recovery after osteotomy is related to the amount of transection, factors such as the occlusion state may be restored to

Correspondence to: Dr. Tadahito Saito, Department of Oral and Maxillofacial Surgery, Nihon University School of Dentistry, 1-8-13 Kanda-Surugadai, Chiyoda-ku, Tokyo 101-8310, Japan. Tel: +81-3-32198102 Fax:+81-3-3219-8355 E-mail: saitou-t@dent.nihon-u.ac.jp normal levels in some cases even if bone restoration has not been performed. When evaluating the process of osteoanagenesis, the impact of various factors such as hematogenic or surrounding histological states and loads should be considered.

The mandible is a membrane bone, and osteoanagenesis and recovery from fractures in this type of bone differ from those in long bones such as the limbs skeleton. Furthermore, the mandibular condyle is a special site that forms a movable joint in a membrane bone, and the regeneration process of this site is distinct from that of other membrane bones.

Although it is known that the periosteum is related to osteoanagenesis in long bones and membrane bones, the extent of participation of the mandibular condyle in bone regeneration is unclear. We propose that the roles of the periosteum and the mandibular condyle in osteoanagenesis should be examined in detail so that these findings can be used in cases in which clinical condylectomy is necessary. 


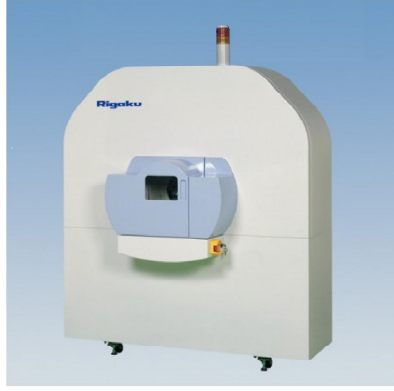

Figure 1. The R-mCT used for exposure (Rigaku Co., Tokyo, Japan).

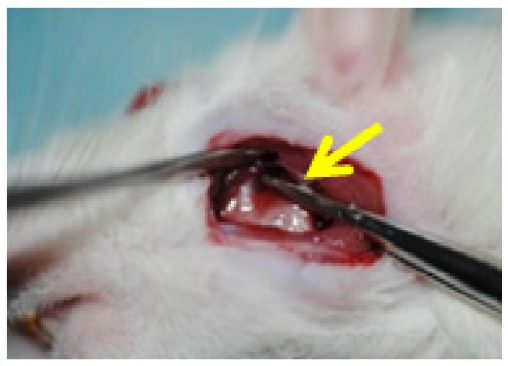

Figure 2. Intraoperative image. The defect site is shown, with the arrow indicating the mandibular condyle.

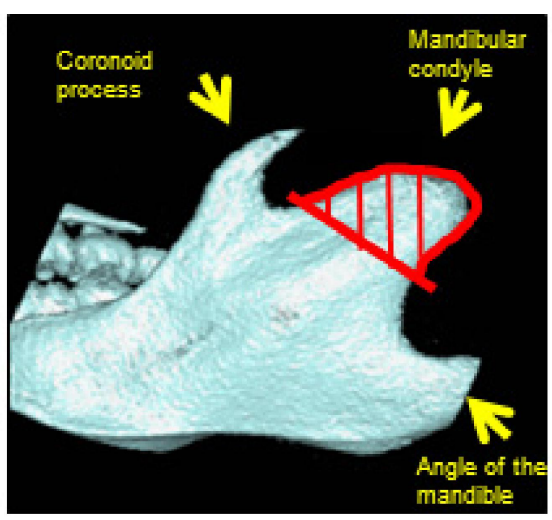

Figure 3. The rat's left mandible is shown from the outside. The schema depicts a surgical site.
Although previous reports have described mandibular-condyle regeneration in animals, few studies have observed osteoanagenesis in the same individual in a chronological manner, and only two-dimensional imaging was performed using radiologic imaging. Therefore, we radiologically analyzed the osteoanagenesis process after condylectomy and performed histological confirmation to evaluate bone restoration over time in the same rat. Furthermore, to investigate the influence of the periosteum on osteoanagenesis, we qualitatively examined the process of mandibular-condyle regeneration in the same individual on three-dimensional imaging by using micro-computed tomography. Finally, the three-dimensional data were analyzed quantitatively.

\section{Materials and Methods}

\section{Study protocol}

Male Wistar rats aged 6 weeks were divided into 2 groups: the periosteum preservation (PP) and periosteum removal (PR) groups. In the PP group $(n=30)$, mandibular condyles were removed up to the mandibular notch, but the periosteum was conserved around the mandibular condyles. In the PR group $(n=15)$, mandibular condyles were removed up to the mandibular notch along with the periosteum. After the surgeries were performed to remove the condyles, bone restoration was observed in these 2 groups.

Images of bone regeneration of the mandibular condyles were obtained immediately after surgery ( 0 weeks) and 1, 2, 3, 4, 6, and 8 weeks later by an in vivo micro X-ray computed tomography (CT) system (R-mCT; Rigaku Co., Tokyo, Japan) (Fig. 1). Longterm observations were also obtained. For qualitative examination of bone regeneration, i-VIEW (Morita Co., Tokyo, Japan) was used for image reconstruction. The 3by4viewer2011 ${ }^{\circledR}$ (Kitasenju Radist Dent, Tokyo, Japan) was used to quantitatively evaluate bone regeneration.

\section{Surgical procedure}

The rats were anaesthetized with intraperitoneal administration of pentobarbital sodium ( $50 \mathrm{mg} / \mathrm{kg}$, Kyoritsu Seiyaku Co., Tokyo,
Japan). The hair on the left side of the jaw was cut from the mandibular condyle to the molar teeth, and an incision was made in the skin in this area parallel to the occlusal plane and expanded for $2 \mathrm{~cm}$ with scissors, exposing the masseter muscle. The masseter muscle was re-anaesthetized with $2 \%$ lidocaine (Xylocaine; AstraZeneca, Osaka, Japan) and was incised approximately 1.5 $\mathrm{cm}$ parallel to the occlusal plane with a surgical knife. The surgical knife reached up to the mandibular bone in depth (Fig. 2).

In the PP group, the masseter muscle and periosteum were detached from the mandibular bone until the mandibular condyle was visible, and the condylar neck and the articular head were subsequently uncovered. The condylar neck was gripped with surgical clips and cut with scissors (Fig. 3). The articular head was removed without its periosteum.

In the PR group, the procedure described above was followed except that the periosteum around the articular head was not detached and the articular head was removed together with its periosteum.

After the condylar head was removed, the wound was sutured with 5-0 nylon threads.

Bone regeneration of the mandibular condyles was observed using an X-ray CT system (R-mCT). The rats were anaesthetized with isoflurane (DS Pharma Animal Health Co. Ltd., Osaka, Japan) and placed into the R-mCT. Radioscopy was performed in the horizontal and vertical directions, and the temporomandibular joint condyles were confirmed to be at the center of the field of the vision.

Bone regeneration images of the mandibular condyles were produced in the sagittal, axial, and frontal planes using micro-CT equipment. The micro-CT exposure conditions were 17 seconds at 6.7 magnification power and $90 \mathrm{kv} / 100 \mathrm{~mA}$. The same monitor was used to create uniform brightness at the time of image evaluations. Bone regeneration images were obtained immediately after surgery ( 0 weeks) and 1, 2, 3, 4, 6, and 8 weeks later. These images were constructed into three-dimensional images using iView. 
Tadahito Saito et al.: Micro-CT Observation

A

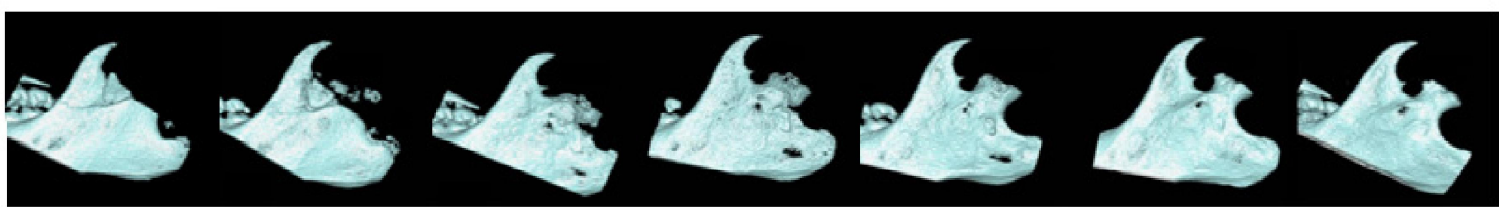

B

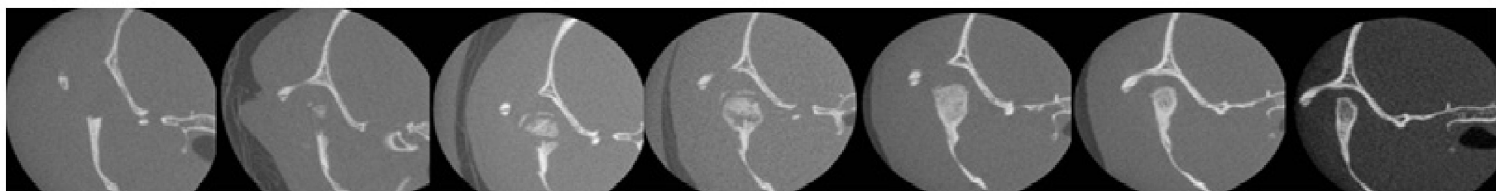

Figure 4. Row A: 3D images from immediately ( 0 weeks) to 8 weeks after surgery in the PP group. Row B: CT images of a forehead incision from immediately ( 0 weeks) to 8 weeks after surgery in the PP group.

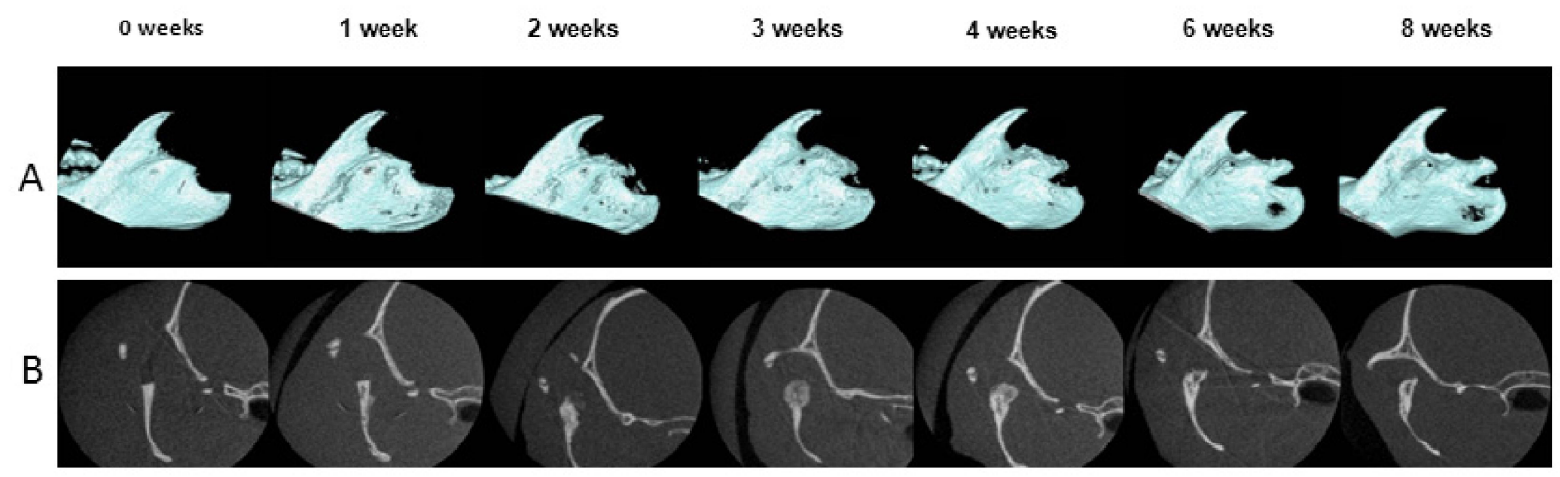

Figure 5. Row A: 3D images from immediately ( 0 weeks) to 8 weeks after surgery in the PR group.

Row B: CT images of a forehead incision from immediately ( 0 weeks) to 8 weeks after surgery in the PR group.

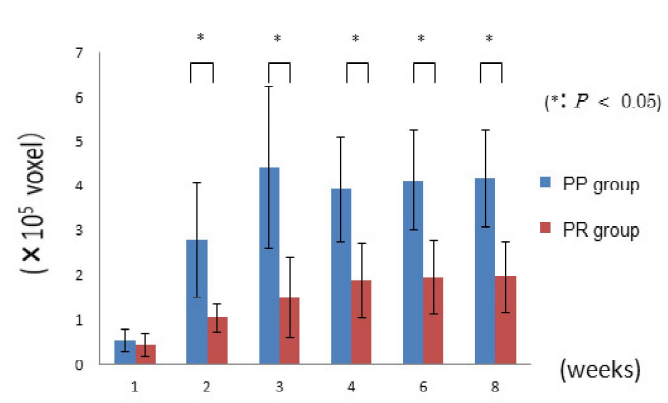

Figure 6. A graph depicting CT data that were analyzed quantitatively using 3by4viewer2011

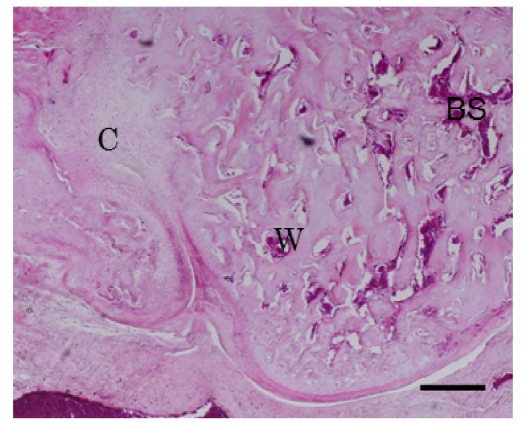

Figure 8. Histology at the fourth week in the PP model. BS: bone stump. C: cartilage-like tissue W: woven bone. Scale bar: $100 \mu \mathrm{m}$

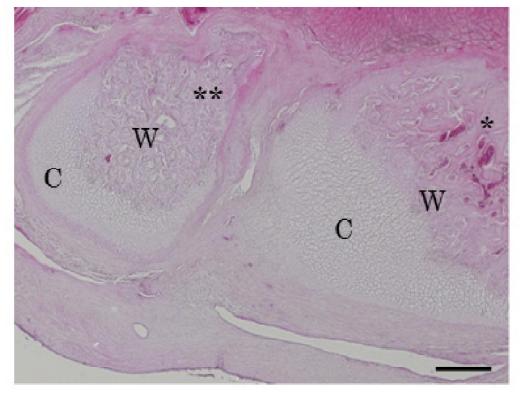

Figure 7. Histology at the second week in the PPmodel. *: bone formation at the bone stump. **: bone formation at the area in which detachment occurred from the bone stump. C: cartilage-like tissue. W: woven bone. Scale bar: $100 \mu \mathrm{m}$

All experiments were approved by the Animal Experimentation Committee at the Nihon University School of Dentistry.

\section{Statistics}

Every week, the F-test was used to test for bone mass variance between the PP and PF groups. A P-value of $<0.05$ indicated that the variance was not equal. Welch's t-test was used to test for the mean value of bone mass every week between the 2 groups. Pvalues $<0.05$ were considered statistically significant. 


\section{Histological staining}

The mandibular bones removed from the rats were fixed in $10 \%$ formaldehyde solution and cut into slices that were large enough to contain the PP site. The slices were subsequently dematerialized in Kalkitox (Wako Pure chemical Industries, Ltd., Osaka, Japan) and embedded in paraffin. The embedded specimens were cut into 8-ìm sections for slide preparation, stained with Hematoxylin and Eosin (HE), and analyzed under an optical microscope.

\section{Results}

\section{Radiological analysis}

In the PP group, bone formation was observed in the bone stump and at the area in which detachment from the bone stump had occurred 1 week after surgery (Fig. 4). The spicule was connected to the bone stump in the second week, and bone mass increased in the direction of the mandibular condyle during the following week. The difference in bone mass between the first and second weeks was conspicuous, and the bone became longer but thinner between the third and fourth weeks, resulting in decreased bone mass. The mandibular condyle became smooth in the fourth week. Although the mandibular condyle increased in the direction of the mandibular fossa up until the sixth week, it did not show remarkable change in the eighth week. In the PR group, bone regeneration was observed in the bone stump in the first week, and bone mass gradually increased thereafter until the sixth week. The peak increase in bone mass that occurred in the PP group was not observed in the PR group. The shape of the mandibular condyle became smooth in the fourth week (Fig. 5). The bone absorption of anterior site of condyle and bone formation of posterior site of condyle was observed in sixth week. The mandibular condyle did not show remarkable change in the eighth week.

Ossification of the mandibular condyle was measured chronologically using micro-CT. In both the PP and PRgroups, the surface layer of the nascent bone was juvenile bone, which exhibited high ossification.

\section{Quantitative analysis}

In the PP group, bone mass increased remarkably from the first week to the third week, but decreased during the fourth week. Thereafter, bone mass increased gradually until the sixth week. The remarkable increase in bone mass that was observed in the PP models did not occur in the PR group, and bone mass increased gradually from the first to sixth weeks in the latter group.

\section{Statistical analysis}

In the PP group, an increase of $2.3 \times 10^{5}$ (voxel) was observed in the average value of increased ossification between the first and second weeks. An increase of $1.6 \times 10^{5}$ (voxel) was observed from the second to the third week, and was reduced to $0.5 \times 10^{5}$ (voxel) from the third to the fourth week.

In the PF group, an increase of $0.6 \times 10^{5}$ (voxel) was observed between the first and second weeks, $0.4 \times 10^{5}$ (voxel) from the second to the third week, and $0.5 \times 10^{5}$ (voxel) from the third to the fourth week.

The bone masses that were measured with the 3by4viewer were evaluated using Welch's t-test in both the PP and PR groups (Fig. 6). Statistically significant differences in bone volume were observed between the PR and PP groups at 2, 3, 4, 6, and 8 weeks $(P<0.05)$.

\section{Histological findings}

In the PP and the PR group, newly formed bones were present at the cleavage site of the condylar neck in all of the preparations, and these bones showed an increase with each chronological preparation.

The formation of an organized structure similar to cartilage was observed in the bone stump from the second week (Fig. 7). Furthermore, we confirmed the formation of woven bone. In the fourth and sixth weeks, we observed a gradual change from cartilagelike tissue to woven bone (Fig. 8).

\section{Discussion}

In this study, we observed the bone regeneration process of the mandibular head after condylectomy. In those cases in which the periosteum was preserved, bone regeneration occurred markedly for up to 4 weeks after condylectomy. However, the bone regenerative volume was reduced after 4 weeks and the maximum value of the newly formed bone was observed at 4 weeks. At 8 weeks, the condylar head was restored to an almost normal state. Although the bone regeneration period was similar among the periosteum-excised cases and the periosteum-preserved cases, the amount of bone regeneration was lower in the periosteumexcised cases.

We have demonstrated reproduction of the mandibular head from the periosteum in the PP group. Several studies have observed the restoration process after mandible defects in various large animals such as sheep and monkeys, among others ${ }^{1-3)}$. In many of these studies, transection of the mandibular head and the muscular process was performed; however, few studies have evaluated only mandibular head defects. Bone lengthening was used in several of the studies in which the mandibular head was transected ${ }^{4,5}$.

Models in which only the mandibular head was removed have been widely studied radiologically using soft X-rays and histological observations, and several studies have measured the mineral quantity of the cortical bone ${ }^{6}$. However, many studies have examined alterations in occlusion caused by transection of the mandibular head ${ }^{6-8)}$, the direction and position of the regenerated mandibular head ${ }^{7,8)}$,or the thickness of the temporomandibular 
Tadahito Saito et al.: Micro-CT Observation

joint disk $^{7}$. Clinical case reports showed that the mandibular head was regenerated and functionally recovered in a case in which only the mandibular head was removed; furthermore, progressive observation was performed at the time of fracture of the mandibular head in a child and at the time of development of an osteochondroma $^{9,10)}$.

Bone reproduction from the periosteum was similar in our study and in the research described above. However, we additionally observed the chronological process of regeneration of the bone from the periosteum by following the same individual using micro CT.

We performed three-dimensional examination of regenerative bone in the same rat by using micro-computed tomography. Until now, several studies have observed defective mandible models using CT, but little research has been conducted with regard to the mechanism underlying a defective mandible head ${ }^{11)}$. In our study, micro-CT the first week after surgery revealed bone regeneration from a distant position that was recognized as a mandibular head transection stump. This is the first report to describe this phenomenon. This observation was regarded as osteoanagenesis from the periosteum in a position distant from the bone stump.

Although an increase in bone mass was observed until the third week and absorption was observed in the fourth week in the PP models, the bone was reproduced as a rough osseous lump until the third week. The bone attained a structural shape near the mandibular head at the fourth week and ripened as an osseous lump. Bone remodeling was observed until the sixth week, but a massive change was not observed thereafter. In the PR model, a gradual increase in bone mass was observed until the sixth week. On the other hand, no difference was observed in the duration of bone formation between the model in which the periosteum was removed and the model in which the periosteum was preserved; however, it was observed that formation occurred from a bone stump in both groups. These processes were observed in threedimensional data. Quantitatively, the PR model also showed a low total amount of bone formation.

In a previous study, a model in which the mandibular head and all of the periosteum were resected did not show reproduction of the mandibular head ${ }^{12)}$. However, although restoration from a bone stump was found in our study, it did not lead to functional recovery ${ }^{12}$. Furthermore, even though a difference was observed in the amount of bone regenerated between the group in which condylectomy with conserved periosteum was performed and the group in which condylectomy with the entire periosteum was performed, we believe that this finding was influenced by bone reproduction from the periosteum.

Histological evaluation revealed that the result of condylectomy in this study was consistent with results of previous reports. We observed cartilage-like tissue in the bone stumps, and this pseudocartilage was carrying out the ossification process in a sequential manner ${ }^{1,7,8,13)}$.

Thus far, in most of the studies that have observed reproduction of bone after creating a defect of the mandible, the defect has been created in the body of mandibible section ${ }^{14-20)}$. Furthermore, several researchers attempted to transplant autogenous bone, allogeneic bone, decalcified powdered bone, and freeze-dried bone ${ }^{14-17)}$. In other studies, the influence of PRP or the effects of Vivosorb ${ }^{\circledR}$, Bio-Gide ${ }^{\circledR}$, and Gore-Tex ${ }^{\circledR}$ have been observed in the defect area ${ }^{12}$, 21, 22).

For studying osteoanagenesis from the periosteum, experiments have been performed in other parts of the body, such as a lower limb, the body of mandibible area, or a rib ${ }^{23-28)}$. Although several researchers used a transplanted periosteum for osteoanagenesis from the periosteum of the mandibular head ${ }^{12,29)}$, these researchers did not observe these factors in the same individual.

Both the method of measuring bone mass and the method of observation described herein can be applied in the model mentioned above, in which artificial material is used in bone defects.

We believe that the present study enabled elucidation of the characteristics or stages of bone production because we observed the chronological process of bone regeneration from the periosteum in the same individual using a rat that does not take time to breed comparatively.

In conclusion, we demonstrated that the bone stump and the periosteum regenerated the mandibular head in which a defect was created by condylectomy. In those cases in which the periosteum was preserved, bone regeneration occurred markedly for up to 4 weeks after condylectomy. At 8 weeks, the condylar head was restored to an almost normal state. Although the bone regeneration period was similar among the periosteum-excised cases and the periosteum-preserved cases, the amount of bone regeneration was lower in the periosteum-excised cases. Our findings suggest that the periosteum is clinically important for reconstructing the mandibular head.

\section{Acknowledgements}

This study was supported by a Grant from the Dental Research Center, Sato Funds, Uemura Fund, and Nihon University School of Dentistry, as well as special research grants for the development of distinctive education for private schools of Japan.

\section{References}

1. Monje F, Delgado E, Navarro MJ, Miralles C and Alonso del Hoyo JR. Changes in temporomandibular joint after mandibular subcondylar osteotomy: an expermental study in rats. J Oral Maxillofac Surg 51(11):1221-1234, 1993

2. Ellis E 3rd, Schneiderman ED and Carlson DS. Growth of the mandible after replacement of the mandibular condyle: an experimental investigation in Macaca mulatta. J Oral Maxillofac Surg 60(12):1461-1471, 2002 
3. Bakker VM, Swartberg PE and Heeley JD. Tissue repair after condylectomy in growing Macaca fascicularis. J Dent Res 63(2):114-119, 1984

4. Zhu SS, Hu J, Ying BB and Li JH. Growth of the mandible after condylar reconstruction using transport distraction osteogenesis: an experimental investigation in goats. Plast Reconstr Surg 121(5):1760-1767, 2008

5. Meng Q, Chen G, Long X, Deng M, Cai H and Li J. Histological evaluation of condylar hyperplasia model of rabbit following distraction osteogenesis of the condylar neck. J Oral Rehabil 38(1): 27-33, 2011

6. Fujita T, Ohtani J and Shirakura M. Changes in cortical bone mineralization in the mouse mandible with regenerated condyle. Eur J Oral Sci 119(2): 136-140, 2011

7. Miyamoto $\mathrm{H}$ and Matsuura $\mathrm{H}$. Unilateral mandibular condylectomy in lambs.Br J Oral Maxillofac Surg 39(4):3049, 2001

8. Açikgöz A. Bilateral bifid mandibular condyle: a case report. J Oral Rehabil 33(10): 784-787, 2006

9. Nishijima K, Kishi K and Nagahata S. Bone regeneration after hemimandibulectomy including mandibular condyle. Journal of the Japanese Stomatological Society 21(4): 491-497, 1975

10. Santiago Vale D, Marotta Araujo M, Cavalieri I, Real Gabrielli M, Pessoa Santos M and Dos Santos Canellas J. Treatment of severe facial asymmetry secondary to osteochondroma of the mandibular condyle: case report. Minerva Stomatol 60(5): 271-278, 2011

11. Ebina H, Hatakeyama J, Onodera M, Honma T, Kamakura S, Shimauchi H and Sasano Y. Micro-CT analysis of alveolar bone healing using a rat experimental model of critical-size defects. Oral Dis 15(4): 273-280, 2009

12. Ueno T, Kagawa T, Fukunaga J, Mizukawa N, Kanou M and Fujii T. Regeneration of the mandibular head from grafted periosteum. Ann Plast Surg 51(1): 77-83, 2003

13. Sakuma $\mathrm{H}$. The histological characteristic of the bone formation in the lower jawbone mandibular head of the Beagle dog. Dental Medicine Research 25: 249-256, 2005

14. Zecha PJ, Schortinghuis J, van der Wal JE, Nagursky H, van den Broek KC and Sauerbier S. Applicability of equine hydroxyapatite collagen (eHAC) bone blocks for lateral augmentation of the alveolar crest. A histological and histomorphometric analysis in rats. Int J Oral Maxillofac Surg 40(5):533-542, 2011

15. Arosarena OA and Collins WL. Defect repair in the rat mandible with bone morphogenic protein 5 and prostaglandin E1. Arch Otolaryngol Head Neck Surg 129(10):11251130, 2003

16. Kajikawa A, Hirabayashi S and Harii K. An experimental study on the growth of condylar cartilage, using a new vascularized mandible heterotopic transplant model.J Oral
Maxillofac Surg 61(2): 239-245, 2003

17. Kaban LB and Glowacki J. Induced osteogenesis in the repair of experimental mandibular defects in rats. J Dent Res 60(7):1356-1364, 1981

18. Miloro M, Haralson DJ and Desa V. Bone healing in a rabbit mandibular defect using platelet-rich plasma. J Oral Maxillofac Surg 68(6): 1225-1230, 2010

19. Pretel H, Lizarelli RF and Ramalho LT. Effect of low-level laser therapy on bone repair: histological study in rats. Lasers Surg Med 39(10):788-796, 2007

20. Issa JP, do Nascimento C, Bentley MV, Del Bel EA, Iyomasa $\mathrm{MM}$ and Sebald W. Bone repair in rat mandible by rhBMP2 associated with two carriers. Micron 39(4): 373-379, 2008

21. Gielkens PF, Schortinghuis J, de Jong JR, Raghoebar GM, Stegenga B and Bos RR.Vivosorb, Bio-Gide, and Gore-Tex as barrier membranes in rat mandibular defects: An evaluation by microradiography and micro-CT. Clin Oral Implants Res 19(5): 516-521, 2008

22. Hoogeveen EJ, Gielkens PF, Schortinghuis J, Ruben JL, Huysmans MC and Stegenga B. Vivosorb as a barrier membrane in rat mandibular defects. An evaluation with transversal microradiography. Int J Oral Maxillofac Surg 38(8): 870875,2009

23. Oriya S. An experimental study on the bone regeneration of mandibular bone defects: The effect on Periosteum. Journal of the Japanese Stomatological Society 31(3): 438-454, 1985

24. O'Driscoll SW, Keeley FW and Salter RB. Durability of regenerated articular cartilage produced by free autogenous periosteal grafts in major full-thickness defects in joint surfaces under the influence of continuous passive motion. A follow-up report at one year. J Bone Joint Surg Am 70(4): 595-606, 1988

25. Ritsilä V, Alhopuro S and Rintala A. Bone formation with free periosteum. An experimental study. Scand J Plast Reconstr Surg 6(1): 51-56, 1972

26. van den Wildenberg FA, Goris RJ and Tutein NoltheniusPuylaert MB. Free revascularised periosteum transplantation: an experimental study. Br J Plast Surg 37(2): 226-235, 1984

27. Takato T, Harii K, Nakatsuka T, Ueda K and Ootake T. Vascularized periosteal grafts: an experimental study using two different forms of tibial periosteum in rabbits. Plast Reconstr Surg 78(4): 489-497, 1986

28. Takato T, Harii K and Nakatsuka T. Osteogenic capacity of vascularised periosteum: experimental study using rib periosteum in rabbits. Br J Plast Surg 41(5):528-532, 1988

29. Ueno T, Kagawa T, Mizukawa N, Nakamura H, Sugahara T and Yamamoto T. Cellular origin of endochondral ossification from grafted periosteum. Anat Rec 264(4): 348-357, 2001, 15(4):273-280, 2009 
